\title{
PHOTOELASTIC INVESTIGATION OF METAL-FORMING PROCESSES USING NEW SAPPHIRE DIES
}

\author{
by S.D.K. Barbat and R.S. Rao
}

\begin{abstract}
Sapphire dies (of single-crystal aluminum oxide) are used to obtain interfacial conditions along the contact surface of the die in a strip-drawing operation. Several solutions based on slip-line field analysis and upper-bound techniques are available for metal-forming problems. However, these solutions generally neglect elasticity and work-hardening and assume that friction is constant along the die-work interface. While these analyses are sufficient for estimating forming loads, they cannot predict residual stresses nor do they accurately define the local stresses and deformations. Furthermore, inadequate information on the frictional conditions at the die-work interface precludes quantitative predictions of the actual forming process.

In earlier research on the drawing of tin-plated mild steel with transparent dies, interfacial velocities were measured experimentally. ${ }^{1,2}$ The velocities were then used as the prescribed boundary conditions in the finite-element analysis to provide complete information on the stresses and strains throughout the deformation zone of the strip. There were some experimental difficulties in obtaining accurate velocity profiles along the die-work interface, especially at the entry and exit regions, due to small contact length.

The photoelastic technique was applied to the study of drawing operations about 30 years ago. Since the dies in those studies were made of photoelastic materials such as epoxy resins and fused quartz, only low-strength, nonstrain-hardening materials such as lead could be drawn., Thus, the earlier work was not representative of commercial materials processing. By contrast, synthetic sapphire (aluminum oxide, $\mathrm{Al}_{2} \mathrm{O}_{3}$ ) is hard enough to machine and form commercial materials. The use of transparent sapphire dies in experimental studies of drawing makes the observation of tribological features at the die-work interface possible during processing commercial materials such as steel, aluminum, and copper. ${ }^{5}$
\end{abstract}

S.D.K. Barbat is Research Assistant and R.S. Rao is Assistant Professor, The University of Michigan, Department of Mechanical Engineering and Applied Mechanics, Ann Arbor, MI.
The moiré interferometry technique was also recently applied to the metal-forming operation. The interfacial conditions were obtained along the contact zone between a hardened tool steel die and a low-carbon steel workpiece in strip drawing. ${ }^{6}$ Rcently, the tangential and normal stresses along the rake face in the orthogonal machining of steel were obtained using the birefringent properties of sapphire.? A similar photoelastic technique was applied to metalforming processes.

In this study, photoelasticity experiments were conducted based on the birefringent properties of sapphire to reveal the isochromatic and isoclinic fringe patterns at the diework interface. Such fringe patterns will eventually be analyzed to obtain the contact stresses (normal and tangential stresses) in the strip-drawing operation.

\section{EXPERIMENTAL PROCEDURE AND EQUIPMENT}

\section{Sapphire Dies and Test Materials}

Synthetic sapphire (aluminum oxide $\mathrm{Al}_{2} \mathrm{O}_{3}$ ) is an important optical material that is grown artificially as a single crystal. In order to grow a single crystal, the aluminum oxide powder is melted in an oxygen/hydrogen flame, and the small molten droplets fall on a pre-selected seed crystal which gradually grows into a large, single-crystal boule. The sapphire is then sliced with a diamond-impregnated saw, machined to size, and ground to the appropriate dimensions to form dies with a semi-drawing angle of $4 \mathrm{deg}$. Each face of the dies is then lapped with diamond compounds. The finished dies are inspected for surface finish, scratches, and surface imperfections. The sapphire dies have a nominal roughness of less than $0.05 \mathrm{~nm}$ on their lapped faces.

Conventional tool and die materials such as tool steel and tungsten carbide are opaque and thus preclude direct observation of the interfacial conditions and the use of some techniques such as photoelasticity to study the boundary conditions quantitatively. The physical and optical properties of synthetic sapphire, however, make it valuable for applications where conventional materials are inadequate. Sapphire has a bulk modulus of $2.07 \mathrm{GPa}$, a modulus of rigidity of $148.5 \mathrm{GPa}$, a modulus of rupture of approximately $551 \mathrm{MPa}$, a Knoop hardness of $1525-2000$, 
and a Young's modulus of approximately $344.5 \mathrm{GPa}$ (all depending on crystal orientation ).

Sapphire can operate satisfactorily at high temperatures and it is chemically inert at temperatures below $1000^{\circ} \mathrm{C}$. It has excellent optical properties such as high transmittance, a low coefficient of thermal expansion, absence of natural birefringence in the 'c' direction (optical axis), and, above all, it exhibits photoelastic properties.

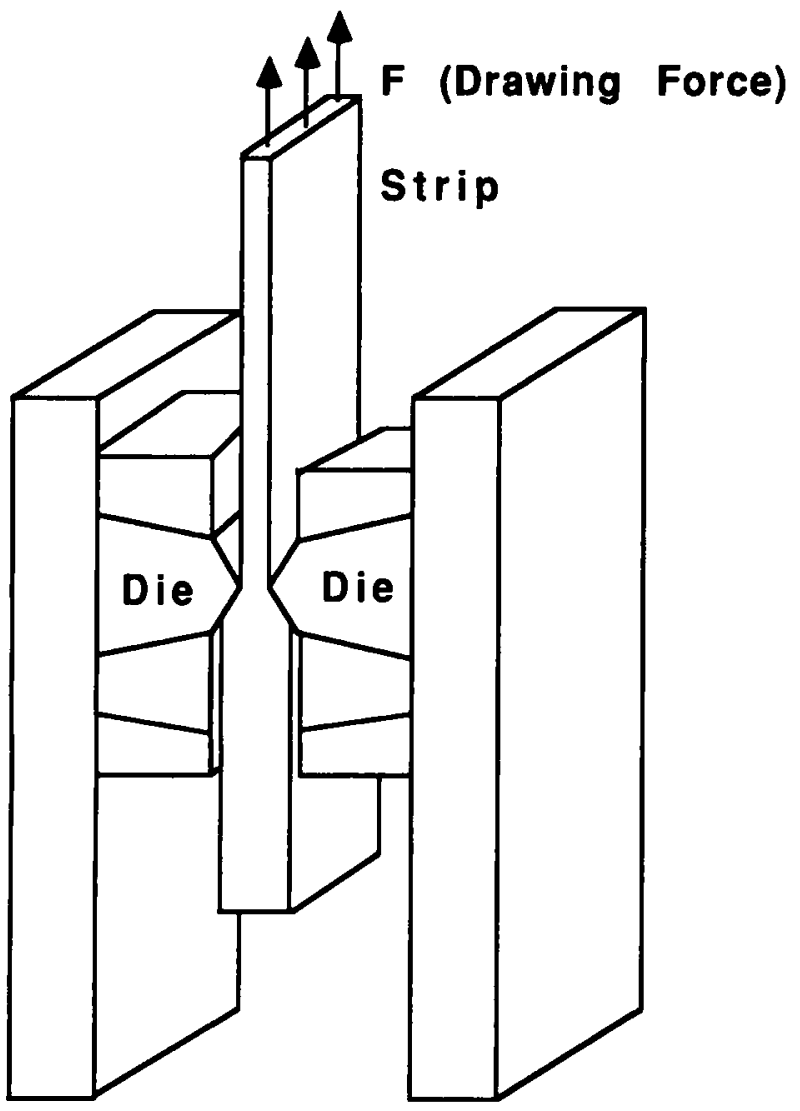

Fig. 1-Simplified layout of the drawing apparatus and dies
It must be pointed out that sapphire is extremely brittle and needs to be handled with care, and that its stress birefringence is relatively weak. Thus, high loads are required to produce a significant number of isochromatics in photoelasticity studies. Sapphire is transparent, exhibits photoelastic properties, and has mechanical properties comparable with those of many tool and die materials such as steels, carbide, and ceramics. Thus, sapphire is an excellent choice as the die material in this study of strip drawing. Commercial materials such as cold-rolled steel, aluminum, and copper are chosen as work materials, and light mineral oil is the lubricant for drawing.

\section{Details of Strip-Drawing Assembly}

The strip-drawing apparatus consists of a pair of identical sapphire dies fixed to the opposing faces of the steel frames. The gap between the dies can be adjusted by means of six gage blocks on the studs, one on each corner and two in the middle. The apparatus is mounted on a microprocessor-controlled Instron testing machine. The drawing speed can be varied from 0.01 to $10 \mathrm{~mm} / \mathrm{sec}$. Figure 1 shows a simplified layout of the drawing apparatus and dies.

\section{Photoelastic Bench}

The lens polariscope setup used in this study is shown schematically in Fig. 2.

\section{The Test}

The strip-drawing apparatus was mounted on a microprocessor-controlled Instron machine. The thickness of a section of the strip was reduced so it could be slipped between the dies. The exit end of the strip was clamped to the moveable cross head, and light mineral oil was used as a lubricant between the dies and the workpiece. The cross section of the strip was initially $13 \times 0.915 \mathrm{~mm}$, and it was reduced in thickness by 28.3 percent to $0.656 \mathrm{~mm}$ while the cross head speed waas maintained at $1.3 \mathrm{~mm} / \mathrm{min}$.

The fringes formed in the die were observed, and increased with the increasing load until they became stable when

Fig. 2-Schematic of polariscope setup

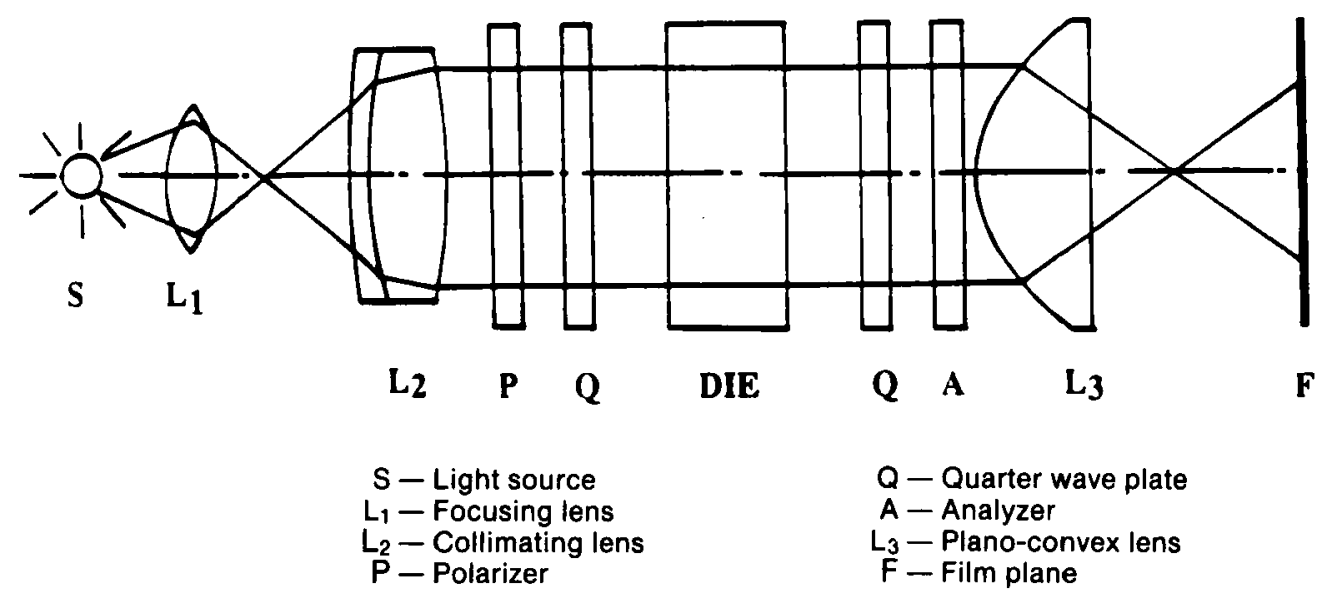




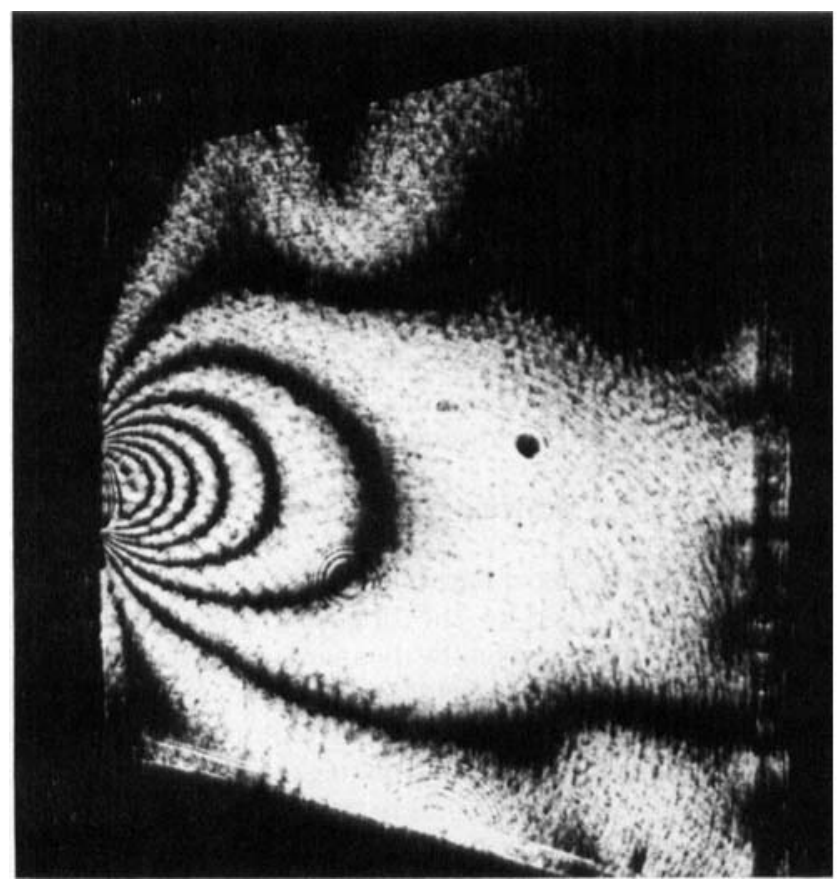

Fig. 3-1sochromatic fringe pattern produced by drawing with sapphire die using a monochromatic light in a dark field

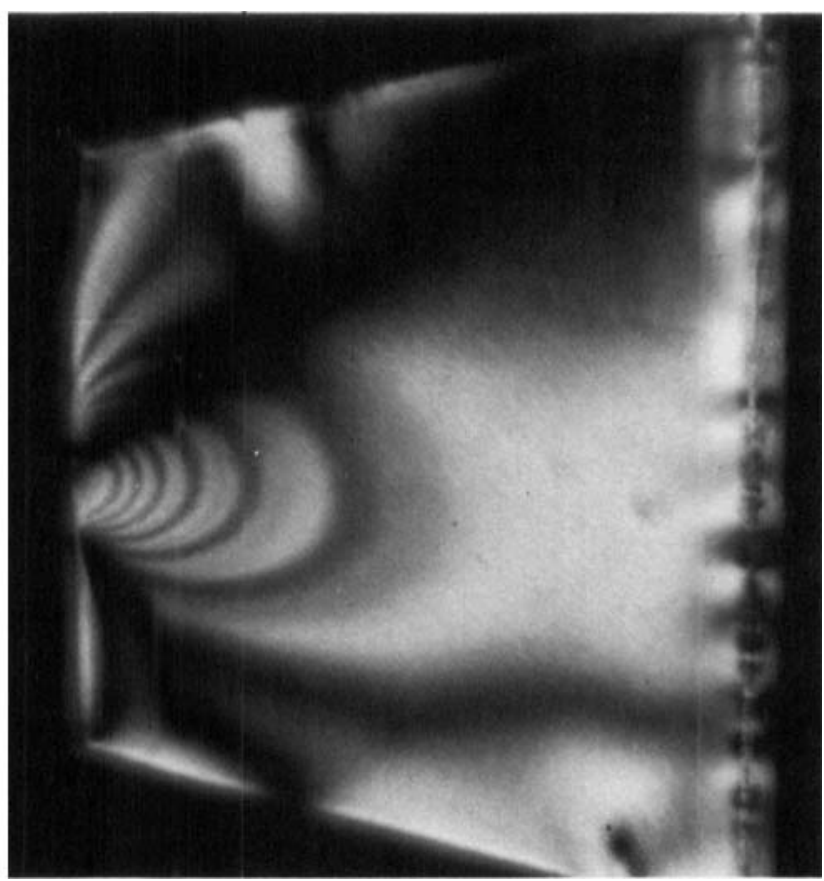

Fig. 4-40-deg isoclinic as diffused dark bands superimposed on color isochromatic obtained from a plane polarized field of white light

a dark background with a monochromatic light in a standard circular polariscope. These fringes represent contours of constant difference between the principal stresses.

Figure 4 represents the $40-$ deg isoclinic, i.e., the locus of all points in the die at which the principal stresses have parallel directions. The 40-deg isoclinic is obtained as diffused dark bands superimposed on the isochromatic color fringes in a plane polariscope using white light. By a systematic rotation of a polarizer and analyzer at 10 -deg intervals, while keeping them crossed, a set of isoclinics was obtained. With these data, the directions of the principal stresses at all points in the die and the principal stress difference at all points can be found.

The traced photographic data of the isochromatic and isoclinic contours are shown in Fig. 5. The stress distribution in the die and along its contact surface with the work material will be found by analyzing these data by using the shear-difference method as described in Ref. 8.

\section{CONCLUSION}

This research reports the first observations and recordings of the isochromatic and isoclinic fringes in sapphire dies during drawing of commercial metals. The fringes in the contact region, between the die and the workpiece, and in its vicinity are crowded and distinguishable. In comparison with previous work, ${ }^{3,4}$ these photoelastic experiments using new sapphire dies are believed to have provided the data closest to the contact region in strip drawing. 


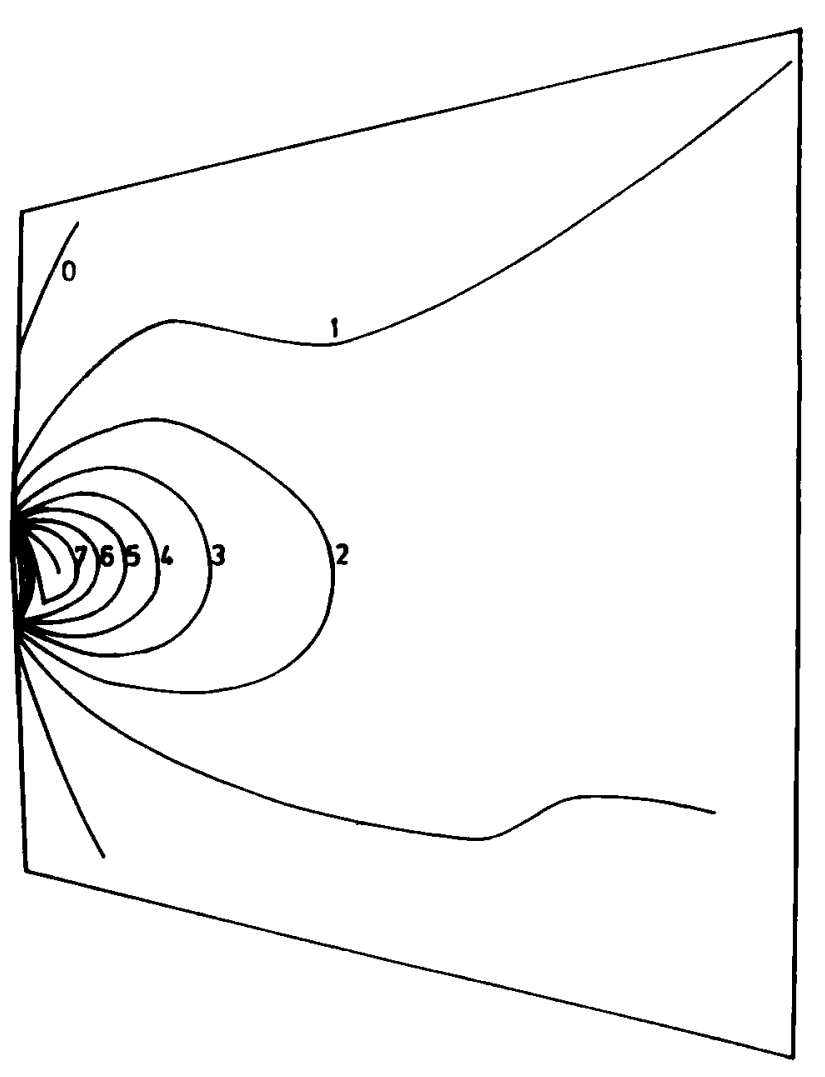

Fig. 58-Isochromatics

Thus, synthetic sapphire is found to be strong enough to withstand loads that arise in processing commercial metals such as low-carbon steels, aluminum, and copper. Transparent sapphire seems to be the only practical material that exhibits birefringent properties suitable for photoelasticity investigation, and its mechanical properties are comparable with conventional die materials such as tool steel and tungsten carbide.

\section{FUTURE WORK}

Several process parameters are now being investigated, and different light sources and lenses are being tested to improve the quality of fringe patterns. Stress distributions in the die will be determined from the isochromatic and isoclinic patterns using the shear-difference method and the model fringe constant. The normal and shear stress components, $\sigma_{\mathbf{x}}, \sigma_{\mathbf{y}}$, and $\tau_{\mathbf{x y}}$ on the contact surface of the die, given by the above analysis, will be used to determine the contact pressure and the frictional stress. Such tractions can lead to the determination of the distribution of the frictional behavior in strip drawing.

\section{ACKNOWLEDGMENTS}

This research work is supported by the National Science Foundation Grant No. DMC-8521744. We thank Professor

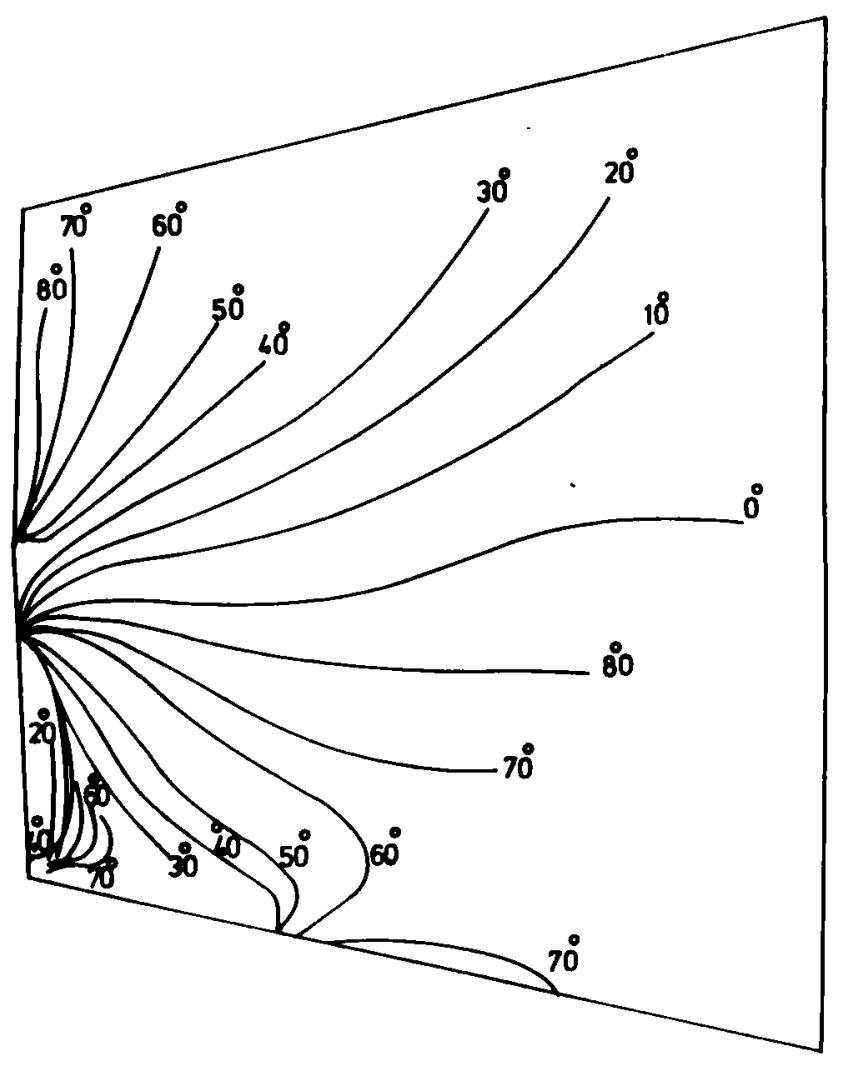

Fig. 5b-/soclinics obtained during drawing a low-carbon steel strip at $1.3 \mathrm{~mm} / \mathrm{min}$ speed for the 28.3-percent reduction

D. Post of Virginia Polytechnic Institute and State University for useful discussions.

\section{REFERENCES}

1. Rao, R.S. Devenpeck, M.L., Wright, P.K., Appleby, E.J., Lu, C.Y. and Richmond, O., "New Strip Drawing Experiments Using Transparent Dies," Int. J. Mech. Sci., 27 (11) and (12), 725-740 (1985).

2. Appleby, E.J., Lu, C.Y., Rao, R.S., Devenpeck, M.L., Wright, P.K. and Richmond, $O$., "Strip Drawing: A Theoretical-Experimental Comparison," Int. J. Mech. Sci, 26 (5), $351-362$ (1984).

3. Ohashi, Y. and Nishikiori, N., "Photoelastic Investigation of Plane Wedge-Shaped Drawing Die, "EXPERIMENTAL MECHANICS, 171-176 (1986).

4. Hemmye, J.H., “An Experimental Technique for the Measurement of Interface Friction in Metal Working, "PhD Thesis, The University of Michigan (1972).

5. Rao, R.S. and Barbat, S.D.K., "Frictional Interactions in Forming Processes: Interfacial Observations and Photoelastic Studies with Transparent Dies, Proc. of Manufacturing Processes, Machines and Systems, "14th Conf. on Production Research and Technology, held at The University of Michigan, October 6-9, 105-108 (1987).

6. Barbat, S.D.K., Guo, Y., Rao, R.S. and Post, D., "Metal-Forming Studies by Moiré Interferometry, "EXPERIMENTAL TECHNIQUES, 2528 (June 1989).

7. Bagchi, A. and Wright, P.K., "Stress Analysis in Machining Using Sapphire Tools," Proc. of The Royal Society of London (1987).

8. Frocht, M.M., Photoelasticity, John Wiley \& Sons,Inc., New York (1), (1941) 